\title{
ASPECTS MORPHOLOGIQUES DE BARTONELLA CANIS
}

\author{
Par le $\mathbf{D}^{\mathrm{r}} \mathrm{J}$. GOYANES
}

Les observations sur la Bartonella bacilliformis et sur la Bartonella muris ratti sont très nombreuses. Il n'en est pas de même avec la Bartonella canis qui, après avoir été trouvée par Kikuth, n'a été retrouvée que par Pérard (1929), Regendanz et Reichenow (1932). Yakimoff et Rastagaieff (1931) ne la trouvent pas chez les chiens splénectomisés de Léningrade, la quatrième observation ayant été signalée par l'école de Whipple (Knutti et Hawkins, Rhoads et Muller, Mac Naught, Woods et Scott).

Nous n'avons pas l'intention de faire la revision de la littérature sur la bartonellose, sur laquelle nous publierons prochainement une revue critique, à laquelle nous renvoyons le lecteur. Dans la note ci-après, nous rendons compte des conditions de notre observation, de l'apparition du parasite et de sa morphologie :

En mars 1935, sept chiens ont été splénectomisés et soigneusement observés au point de vue hématologique, en réalisant des examens répétés de la sécrétion gastrique afin de démontrer l'influence de la splénectomie sur le suc gastrique et l'action subséquente d'un extrait splénique sur ladite sécrétion de chiens splénectomisés.

Chien $\mathrm{N}^{\circ} 1$, splénectomisé le 13-3-1935. Les Bartonella apparaissent le 3-4-1935 (21 jours). Chez ce chien apparut une baisse très marquée du chiffre d'hématies et de l'hémoglobine. En même temps, on observa dans les hématies une inclusion baciliforme granuleuse qui fut identifiée avec une Bartonella (Bartonella canis, Kikuth 1928), car ses caractères structuraux, sa situation et le cadre clinique de la maladie du chien correspondaient à ceux décrits par Kikuth, Regendanz et Reichenow, Knutti et autres. Cet animal, après une longue période de fièvre, fut hématologiquement guéri ;' les Bartonella disparurent brusquement et il ne lui resta qu'une anémie de laquelle il se remit plus tard avec des injections d'un extrait splénique préparé avec une technique semblable à celle recommandée par Whipple dans l'obtention de son extrait hépatique $\mathrm{n}^{\circ} 55$.

Annales de Parasitologie, $\mathrm{T}$ XIV, $\mathrm{N}^{\circ} 5 .-1^{\mathrm{er}}$ septembre 1936. 
Chien $N^{\circ} 2$, splénectomisé le 2-4-1935. Les Bartonella apparaissent le 7-5-1935 ( 35 jours). Ce deuxième chien montra aussi spontanément une bartonellose aiguë, à cours beaucoup plus grave que celui du chien précédent, avec fièvre et hématies parasitées très nombreuses. Au bout de plus de 20 jours, les Bartonella disparurent brusquement. Cependant, l'observation très soignée et répétée et la goutte épaisse, nous permirent de trouver quelques parasites isolés. Le chien se remit lentement de son anémie, mais pas tout à fait.

Chien N ${ }^{\circ} 3$, splénectomisé le 2-4-1935. Bartonella + piroplasmes le 17-5-1935. Ce troisième chien tomba malade avec un cadre encore plus grave que le précédent. Il présentait une infection mixte de Bartonella et Babesia. La fièvre et la gravité clinique se prolongèrent pendant sept jours durant lesquels l'animal fut triste, ne mangea pas et resta couché. Le huitième jour, l'animal chancelait, il ne marchait pas spontanément; le lendemain, les Bartonella avaient brusquement disparu; l'animal était beaucoup mieux, quoique les piroplasmes persistassent. Cette infection fut bénigne, il n'eut jamais de crise d'hémoglobinurie, les parasites ne furent jamais très abondants dans le sang et ils disparurent tout à fait au bout de quelques jours. Malgré tout, l'animal resta anémique : 2.000.000 hématies environ.

ChiEn N ${ }^{\circ} 4$, splénectomisé le 30-3-1935. Bartonella + piroplasmes le 14-6-1935 (76 jours). Chez ce quatrième chien, l'anémie apparut soudain, avec invasion très abondante de piroplasmes et Bartonella, en même temps que l'anémie s'accentuait. L'animal mourut spontanément et en ce moment il atteignait le chiffre de 650.000 hématies avec 25 p. 100 d'hémoglobine et pratiquement avec la totalité de ses hématies parasitées par des Bartonella. Au cours de la maladie, l'animal ne mangea pas, maigrit considérablement, but beaucoup et eut une fièvre considérable. Dans l'autopsie, on apprécia une lésion dégénérative hépatique semblable à celle observée chez les rats. Foie jaunâtre-rougeâtre, avec des régions à dégénération graisseuse. Cœur pâle, flasque (en diastole). Reins congestionnés avec des lésions tubulaires manifestes, cylindres épithéliaux et hématies.

Chien N ${ }^{\circ} 5$, splénectomisé le 31-3-1935. Bartonella + piroplasmes le 10-5-1935 (40 jours). Ce cinquième chien présenta de la fièvre avec anémie intense sans hémoglobinurie. L'examen du sang montra l'existence d'une petite quantité de Bartonella qui disparurent brusquement ; on y voyait aussi des piroplasmes en quantité réduite 
et on observa des rosettes avec jusqu'à 8 éléments piriformes. Quelques jours avant la mort les Bartonella disparurent rapidement et plus tard les piroplasmes. L'animal mourut avec le sang libre de parasites.

ChiEn ${ }^{\circ} 6$, ne fut pas splénectomisé. Les piroplasmes apparurent le troisième jour de son arrivée à notre chenil. Ce sixième chien, avec rate, subit une infection légère de piroplasmes, spontanément

\begin{tabular}{|c|c|c|c|c|c|c|c|}
\hline Chiens $N^{\circ}$ & 1 & 2 & 3 & 4 & 5 & 6 & 7 \\
\hline $\begin{array}{l}\text { Spléneclomie... } \\
\text { Bartonella..... } \\
\text { Babesia........ } \\
\text { Bartonella Ba- } \\
\quad \text { besia........ }\end{array}$ & $\mid \begin{array}{r}13-3-35 \\
3-5-35 \\
\end{array}$ & $\mid \begin{array}{l}2-4-35 \\
7-5-35\end{array}$ & $2-4-35$ & $30-3-35$ & $31-3-35$ & $17-5-35$ & $22-3-35$ \\
\hline
\end{tabular}

guérie. Il eut une crise d'hémoglobinurie avec perte d'appétit, somnolence, amaigrissement, etc. Le parasite disparut rapidement du sang. Toutefois, pendant un certain temps, une anémie discrète continua. Il ne nous a pas été possible de découvrir en aucun moment des Bartonella dans le sang de cet animal.

Chien N ${ }^{\circ} 7$, splénectomisé. Ce chien ne présenta aucune infection malgré les inoculations (1).

Les brèves histoires cliniques que nous exposons montrent la fragilité organique produite par la perte de la rate, suivie dans deux cas de mort. Dans le quatrième cas la mort est déterminée par une infection de Bartonella, et dans le cinquième chien par une infection mixte produite par des Bartonella associées à des piroplasmes, pareillement au cas observé par Kikuth.

En 1928, Kikuth décrit les Bartonella comme des bâtonnets de forme et longueur diverse, en arc de violon, doubles bâtonnets, haltères et Coccus. Reichenow et Regendanz ne considèrent pas très heureuse cette description des formes bacillaires qui, à leur avis, ne sont que des chaînettes de Coccus, l'élément initial étant ces formes coccoïdes, à partir desquelles les colonies de Bartonella s'originent à

(1) Pendant la dernière période, plus de 40 chiens sont passés par notre chenil, Pas un seul ne fut infecté, ni spontanément, ni expérimentalement. 
la surface des hématies. Ces auteurs distinguent aussi, dans la forme initiale coccoïde, deux variétés : une granulaire de couleur homogène et une autre plus grande, dont la coloration plus accentuée des bords du parasite lui donne l'apparence d'un anneau. Entre ces deux formes, on trouve des formes transitoires et les auteurs croient pouvoir expliquer la forme annulaire par l'existence d'une couche ou capsule de substance non colorable avec des phénomènes de Quellung. Le Coccus, en se développant, forme une chaînette qui se désintègre et envahit de nouvelles hématies. Dans certains cas, ces auteurs observent quelques formes bacillaires à l'intérieur desquelles l'élément coccoïde apparaît différencié. Ces mêmes formes bacillaires sont aussi observées dans certaines microphotographies. Les auteurs admettent qu'en certains cas les chaìnettes trouent les hématies.

Les observations conduites par Pérard sont d'accord avec celles réalisée par Kikuth.

Les auteurs américains décrivent des formes bacillaires observables d'ailleurs dans leurs microphotographies. Rhoads et Miller trouvent des formes en chaînettes avec ses éléments séparés ainsi que des bacilles simulant des files de Coccus et des filaments avec une "crête protoplasmique » ondulante dans l'un de ses bords. Le filament au total atteint les $6 \mu$. Les formes ovales ont de 0,2 à $0,4 \mu$.

Les auteurs observent des formes libres dans le liquide environnant. Kikuth et Hawkins observent des formes en chapelet reposant radialement sur l'hématie, dans laquelle on remarque vers les bords du parasite deux vacuoles claires. Les formes en chapelet peuvent exister en nombre d'une à quatre sur une seule hématie, à côté de formes coccoïdes. Ces dernières peuvent être très nombreuses sur les hématies ou exister isolées. On observe rarement des formes semilunaires ou en arc de violon. Les formes annulaires sont plus abondantes à proximité de la crise hémolitique qui a lieu vers le troisième-cinquième jour de l'apparition du parasite dans le sang.

Morphologiquement, nous avons observé que les Bartonella se localisent dans la surface des hématies. En certains globules, les éléments de la Bartonella apparaissent sur le bord de l'hématie comme une formation granuleuse qui dépasse la limite de la cellule ou, plus fréquemment, comme un ou deux Coccus collés au bord de la cellule. Dans la plupart des cas, la Bartonella se localise dans la portion centrale de l'hématie.

Dans les formes aiguës très graves (cas 4 et 5), on voit avec une certaine profusion des hématies parasitées avec des formes coccoïdes isolées ou multiples; dans ces cas on trouve des hématies avec 5 
ou 6 longs filaments recouvrant la surface du globule. Dans certains cas on observe aussi des formes filamenteuses en trépied, c'est-àdire, trois filaments avec un point de départ commun, et entre eux il $\mathrm{y}$ en a qui présentent une formation véliforme de laquelle nous

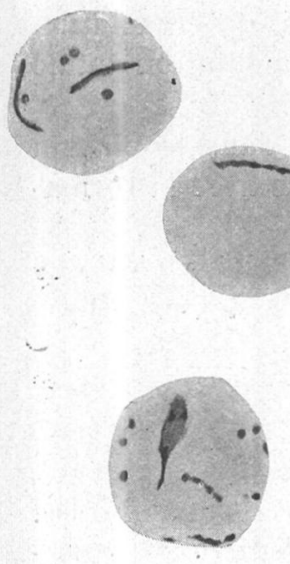

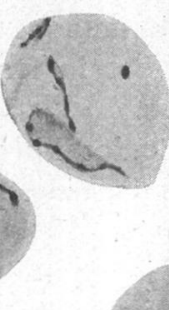
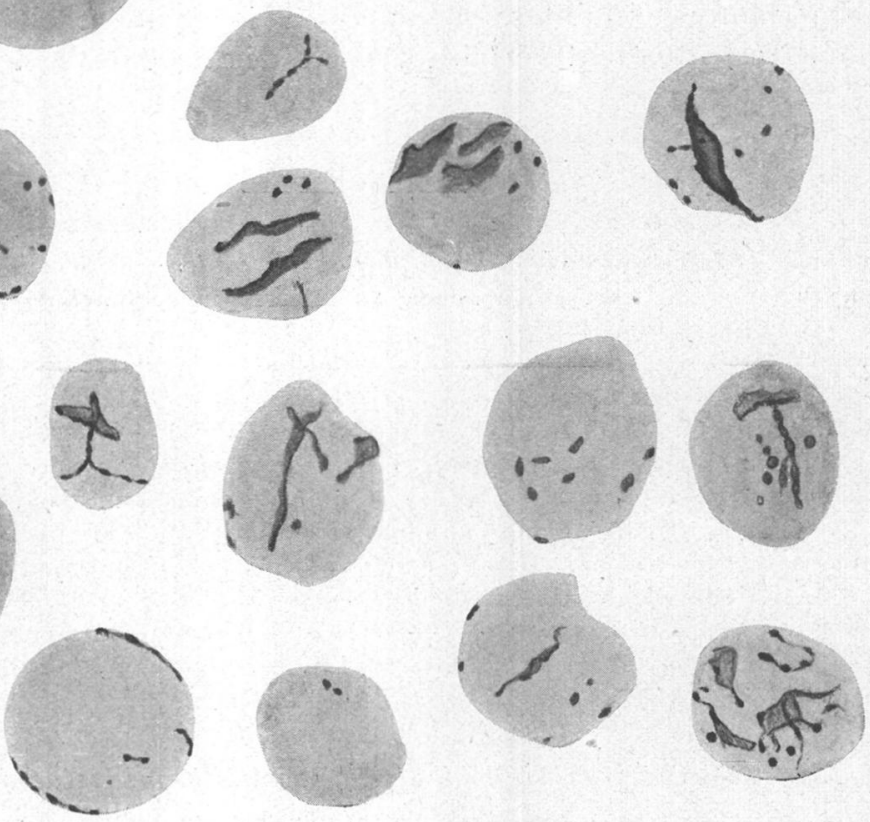

Fig. - Différents aspects morphologiques de Bartonella canis

parlerons plus tard. Dans d'autres hématies les filaments dessinent des figures semblabes à celles de petites branches de mousse.

Les formes en anneau décrites par Reichenow et Regendanz ont, à notre avis, un stade antérieur dans lequel dans la forme filamenteuse-granuleuse on aperçoit une zone à la manière d'une membrane ondulante (libre ?) faiblement teinte avec les colorants de la chromatine. Cette prolongation forme la semilune ou l'arc de vio- 
lon décrit par Kikuth et elle finit par condenser une formation granuloïde sur ce bord.

La forme cocoïde est le produit de la fragmentation d'un Coccus ou filament. Ce dernier est originé à son tour par croissance et segmentation d'un Coccus qui parasite un nouveau globule. L'existence de longs filaments avec une crète véliforme et sa différenciation postérieure en granules semblent indiquer la fragmentation totale de ces éléments filamentaux en coccoïdes.

Nous avons observé des formes coccoïdes libres dans le plasma ainsi que des chaînettes de 5 et 6 éléments, mais pas avec la formation véliforme dont nous avons déjà parlé.

Les figures ci-jointes, dessinées à la chambre claire, à 1.800 diamètres, illustrent certains aspects décrits dans le texte.

\section{Bibliographie}

Perard. - Infection du chien par Bartonella canis. C.R. Soc. Biol., 1929, 100. Kıитн (W.). - Ueber einen neuen Anämieerreger Bartonella canis nov. spec. Klin. W.och., 1928, 7, 1729 .

KNUTTI (R. E.) et Hawkins (W. B). - Bartonella incidence in Splenectomized bile fistula Dogs. Journ. Exp. Med., 1935, 61, 115.

MaG NaUght (J. B.), Woods (F. M.) et Scott (T. V.). - Bartonella bodies in the blood of a non-splenectomized dog. Journ. Exp. Med., 1935, 62, 353.

Regendanz (P.) et Reichenow (E.). - Beitrag zur Kenntnis von Bartonella canis. Arch. f. Schiff. u. Trop. Hyg., 1932, 36, 305.

Rhoads et Pry Miller (D. K.). - The association of Bartonella bodies with induced anemia in the dog. Journ. Exp. Med., 1935, 61, 139.

Yakimov (W. L.) et RastagaiefF. - Sur la Bartonellose des chiens en Russie. Bull. Soc. Path. Ext., 1931, 24, 471.

Laboratoire de Parasitologie de la Faculté de médecine de Madrid (Directeur: Prof. G. Pittaluga). 